\title{
Abelhas evitam flores com modelos artificiais de aranhas
}

\author{
Nayara Carvalho ; ;osué Raizer ${ }^{2}$; Augusto Cesar de Aquino Ribas ${ }^{1, ®}$ \& Milena Delatorre ${ }^{1}$ \\ 1. Programa de Pós Graduação em Ecologia e Conservação. Centro de Ciências Biológicas e da Saúde, Universidade Federal de \\ Mato Grosso do Sul, Campo Grande, MS, Brasil. 2. Faculdade de Ciências Biológicas e Ambientais, Universidade Federal da \\ Grande Dourados, Brasil.
}

\begin{abstract}
REsumo. Aranhas caçando sobre flores podem mudar o comportamento de polinizadores e, em consequência, alterar o sucesso reprodutivo das plantas. No entanto, ainda não é perfeitamente conhecido como diferentes grupos de polinizadores reagem à presença de aranhas e em quais circunstâncias ocorre redução no sucesso das plantas. Neste estudo, avaliamos a frequência de visitas florais e o comportamento de abelhas Apis mellifera L. (Apidae) frente ao risco de predação simulado por modelos de aranhas feitos de resina epóxi. A presença destes modelos reduziu o número de visitas em flores de macrófitas aquáticas Ludwigia tomentosa (Cambess.) (Onagraceae) e aumentou a proporção de comportamentos de refugo, em que as abelhas abandonavam repentinamente as flores sem acessar seus nectários. Este resultado sugere que abelhas percebem traços morfológicos de predadores sobre flores, reconhecendo-as como recurso de baixo valor em função do risco de serem predadas.
\end{abstract}

[Palabras chave: Apis mellifera, camuflagem, interação indireta, predação]

\begin{abstract}
Aвstract. Bees avoid flowers with artificial models of spiders: Spiders hunting on flowers can change pollinators' behavior and therefore change the reproductive success of plants. However, it is not still completely known how different groups of pollinators react to the presence of spiders and in what circumstances there is a reduction in the plants' success. In this study, we evaluated the frequency of floral visits and Apis mellifera L. (Apidae) behavior against the risk of predation simulated by spiders models made of epoxy resin. The models presence has reduced the number of visits to Ludwigia tomentosa (Cambess.) (Onagraceae) flowers and increased the proportion of refusing behavior in which bees suddenly abandoned the flowers without accessing the nectaries. This result suggests that bees perceive morphological traits of predators on flowers, recognizing it as a low value source due to the risk of being predated.
\end{abstract}

[Keywords: Apis mellifera, crypsis, indirect interaction, predation]

\section{INTRODUÇãO}

Alguns predadores insetívoros com estratégia senta-e-espera utilizam flores entomófilas como sítio de forrageamento, as quais atraem polinizadores que passam a ser presas potenciais (Dukas \& Morse 2005). Entre os predadores insetívoros que frequentemente forrageiam sobre flores e fazem uso de tal estratégia estão mantídeos, fimatídeos e aracnídeos (Halaj \& Wise 2001). Muitos deles têm habilidade para desenvolver semelhanças com as flores ou partes florais o que facilita a camuflagem com o substrato (Chittka 2001; Théry \& Casas 2002).

Aranhas Thomisidae são predadores comuns em flores que utilizam de sua camuflagem para emboscar insetos com suas pernas dianteiras raptoriais (Morse 2007; Rocha-Filho \& Rinald 2011). Aranhas desta família escolhem seus sítios de forrageamento avaliando estímulos

Programa de Pós Graduação em Ecologia e Conservação. Centro de Ciências Biológicas e da Saúde, Universidade Federal de Mato Grosso do Sul, 79070-900, Campo Grande, MS, Brasil. ribas.aca@gmail.com táteis, visuais ou ainda odores, que são atrativos para outros visitantes florais (Heiling et al. 2004).

A presença de aranhas sobre flores pode reduzir o número de visitas quando os visitantes evitam flores ao reconhecer um risco potencial de predação pela avaliação de diversas características (Stoks et al. 2003). Tais características podem ser a simetria floral, a presença de coespecíficos (Wignall et al. 2006), traços morfológicos (Freitas \& Oliveira 1996), agressividade (Ness 2006) e odores dos predadores (Dicke \& Grostal 2001).

No Pantanal, um exemplo de planta que possui flores utilizadas como sítio de forrageamento por espécies de aranhas Thomisidae é o arbusto Ludwigia tomentosa (Cambess.) Onagraceae. As plantas desta espécie são macrófitas aquáticas de hábito anfíbio, que podem modificar completamente

Editor asociado: Alejandro Farji-Brener

Recibido: 16 de enero de 2012; Fin de arbitraje: 7 de marzo; Revisión recibida: 13 de abril; Aceptado: 22 de junio. 


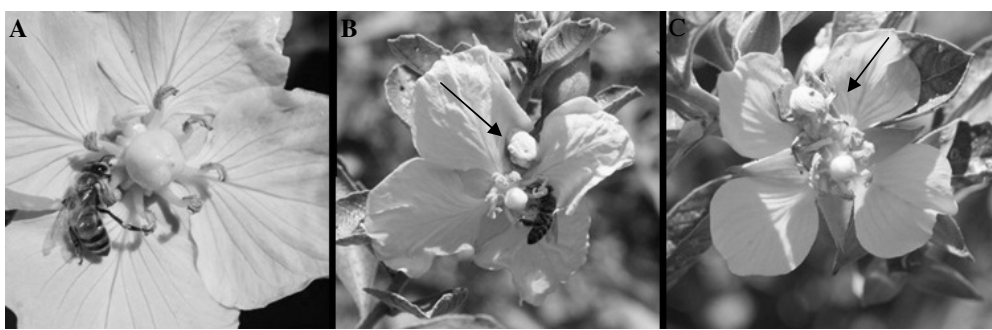

Photos: Nathalia Yurika Kaku-Oliveira (Universidade Federal do Paraná).
Figura 1. Flores de Ludwigia tomentosa que receberam ou não modelos de aranha em resina epóxi formando um gradiente de risco de predação: sem modelo (A), com modelo do opistossoma da aranha (B) e com modelo em resina epóxi e grampos metálicos simulando prossoma, opistossoma e pernas de aranha (C). Em A e B, uma abelha Apis mellifera acessa nectário na base da corola.

Figure 1. Ludwigia tomentosa (Onagraceae) flowers that either received or did not receive an epoxy resin model spider, forming a gradient of predation risk: without a model (A), spider opistossoma model (B) and model with metal clips, simulating prosome, opistossoma and spider legs (C). In A and B a bee Apis mellifera (Apidae) access the nectary at the base of the corolla.

sua morfologia e anatomia entre condições de alagamento e seca. Na sua forma arbustiva em condições de seca, atinge de 1 a 3 m de altura. Florescem quase o ano todo, menos nos meses muitos secos (Pott \& Pott 2000). Suas flores, em formato de prato e de cor amarela, são sítios de forrageamento de aranhas e atrativas para visitantes florais. Um de seus visitantes mais frequentes é a abelha Apis mellifera L. (Apidae), muito abundante na região.

Neste estudo, buscamos compreender como o risco de predação, simulado com modelos artificiais de aranhas, altera a frequência de visitas de abelhas $A$. mellifera a flores $L$. tomentosa. Acabamos por demonstrar que esta simulação de risco de predação modifica o comportamento de visita das abelhas, que tendem a abandonar flores com aranhas.

\section{MÉTODOS}

O Pantanal é uma vasta planície alagável (aproximadamente $148000 \mathrm{~km}^{2}$ ) situada no centro da América do Sul (Harris et al. 2005). O trabalho foi desenvolvido em uma mancha da macrófita aquática L. tomentosa ao longo da Estrada Parque (Rodovia MS 184), Pantanal do Rio Negro, Mato

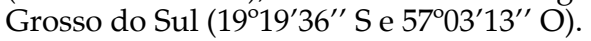

Para simular a presença de aranhas sobre flores L. tomentosa, foram construídos modelos artificiais com resina epóxi (simulando prossoma e opistossoma) e grampos metálicos (simulando pernas), imitando traços morfológicos de aranhas que forrageiam sobre flores (Gonçalves-Souza et al. 2008; Rocha-Filho \& Rinald 2011). Após a confecção destes modelos, foram escolhidas flores buscando homogeneizar a atratividade e a disponibilidade de recompensas florais. Em 27 blocos com três flores cada, foi sorteada uma flor para receber um modelo de aranha, outra um modelo de opistossoma e na flor restante nenhum objeto foi acrescido (Figura 1). Entre os dias 22 e 24 de setembro 2008 foram observados diariamente nove destes blocos, registrando o número de visitas por abelhas $A$. mellifera e de comportamentos de refugo nestas visitas. O refugo consistiu no abandono repentino da flor por parte da abelha, antes de acessar o nectário na base da corola. Cada bloco amostral foi inspecionado por 20 minutos, em dois períodos de 10 minutos ao logo da manhã, período no qual os visitantes estavam mais ativos. Após o primeiro dia, foram amostrados grupos de flores distantes aproximadamente $50 \mathrm{~m}$ do grupo de flores amostrado no dia anterior.

Foram feitas análises de variância para medidas repetidas em três dias, considerando-se o efeito dos tratamentos (flor sem modelo, com modelo de opistossoma ou com modelo de aranha) nos blocos. As variáveis dependentes foram o número de visitas e a proporção de refugos nestas visitas. A proporção de refugos foi transformada para o arcoseno da sua raiz quadrada (Zar 2009). Utilizamos a linguagem R (R Development Core Team 2012) para construir os gráficos, modelos e testes de hipóteses.

\section{RESUlTADOS}

Observamos 883 visitas de abelhas $A$. mellifera, das quais 205 com comportamento de refugos, em que a abelha pousava na flor, mas a abandonava repentinamente sem acessar o nectário. Tanto o número de visitas quanto a frequência de comportamentos de refugo responderam ao gradiente de risco de predação, composto por flores sem modelos de aranhas, flores com modelos de opistossoma de aranhas e flores com modelos de aranhas (Figura 2).

O número de visitas foi maior em flores sem adição de modelos, mas esta variação foi fortemente influenciada pelo dia de amostragem (Tabela 1A), sendo maior no terceiro dia (Figura 2). A proporção de abelhas que refugaram a visita foi maior na presença do modelo de aranha independentemente do dia de amostragem (Tabela 1B), sendo menor no terceiro dia (Figura 2). 


\section{DISCUSSÃO}

As visitas de $A$. mellifera a flores parecem depender da simetria visual da flor que, quando quebrada pela presença de um predador indica um recurso de baixo valor (Leonard et al. 2010). Provavelmente a presença do modelo de aranha diminui a frequência de visitação, pois seu formato de predador efetivo altera a aparência da flor mais do que o modelo de opistossoma, sem os traços morfológicos de predador (Gonçalves-Souza et al. 2008; Jones \& Dornhaus 2011).

Abelhas podem avaliar o padrão de simetria da flor à distância (Leonard et al. 2010) e detectar predadores camuflados (Abbott 2010; Defrize et al. 2010). Com a aproximação da abelha à flor, as aranhas podem ser eficientemente detectadas (Defrize et al. 2010) e a abelha pode desistir de completar a visita. Após a escolha da flor, o visitante já despendeu tempo e energia em busca de seu recurso. Portanto, o comportamento de refugar flores antes de acessar os nectários ocorre em reposta à percepção do predador por abelhas A. mellifera, o que corrobora com o sistema de reconhecimento predador-presa entre tomisídeos e himenópteros (e.g., Dukas 2001; Suttle 2003).

As visitas de abelhas em flores com modelos de aranhas demonstram que a estratégia de forrageamento adotada pelas aranhas
Tabela 1. Análises de variância de medidas repetidas em três dias, para um desenho experimental em 27 blocos de três flores Ludwigia tomentosa no Pantanal do Rio Negro. Cada flor de um bloco recebeu ou não um modelo de aranha em resina epóxi, compondo um gradiente de risco de predação: flor sem adição de modelo, flor com modelo de opistossoma de aranha e flor com modelo de aranha (prosso ma+opistossoma+pernas). Em A, análise para número total de visitas florais por abelhas Apis mellifera (Apidae). Em B, análise para proporção do número de refugos nestas visitas (transformada para arcoseno da raiz quadrada).

Table 1. Analysis of variance with repeated measures for three days, an experimental design in 27 blocks of three flowers Ludwigia tomentosa in the Pantanal of Rio Negro. Each flower block either received or did not receive an epoxy resin model spider, forming a gradient of predation risk: flower without adding model, flower with opistossoma and flower with spider model (proso me+opistossoma+legs). In A, analysis for total number of floral visits by honey bees Apis mellifera. In B, ratio analysis for the number of rejects these visits (arccosine of the square root transformed).

\begin{tabular}{lcccccc}
\hline & \multicolumn{3}{c}{ A - Visitas } & \multicolumn{3}{c}{ B - Refugos } \\
Erro: Entre & gl & F & $P$ & gl & F & $P$ \\
\hline Tratamentos & 2 & 13.2 & $<0.001$ & 2 & 105.4 & $<0.001$ \\
$\quad$ Blocos & 8 & 1.7 & 0.171 & 8 & 3.23 & 0.022 \\
$\quad$ Resíduo & 16 & & & 16 & & \\
Erro: Dentro & & & & & & \\
$\quad$ Dias & 2 & 54.5 & $<0.001$ & 2 & 6.57 & 0.004 \\
$\quad$ Blocos & 16 & 4.1 & $<0.001$ & 16 & 1.57 & 0.13 \\
$\quad$ Tratamentos: & & & & & & \\
$\quad$ Dias & 4 & 0.2 & 0.9 & 4 & 1.17 & 0.34 \\
$\quad$ Resíduo & 32 & & & 32 & & \\
\hline
\end{tabular}

Thomisidae é bem sucedida (Romero \& Vasconcellos-Neto 2005). Essas aranhas são comuns em plantas (Rocha-Filho \& Rinald
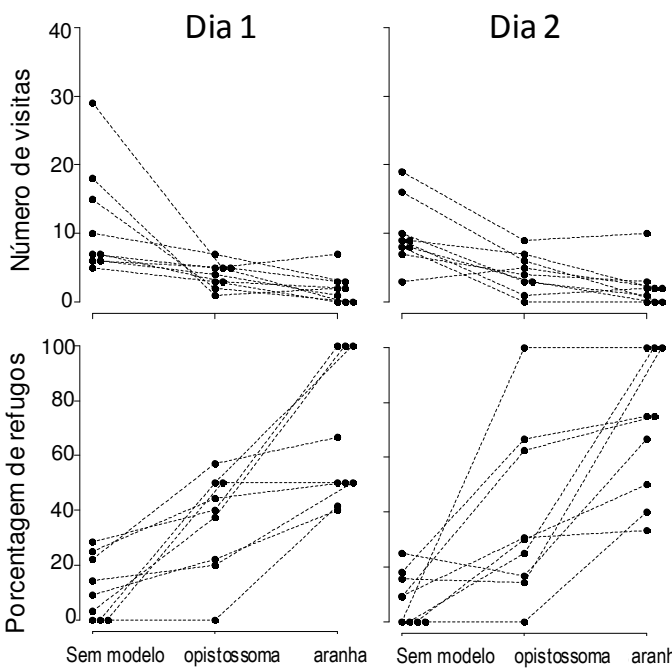

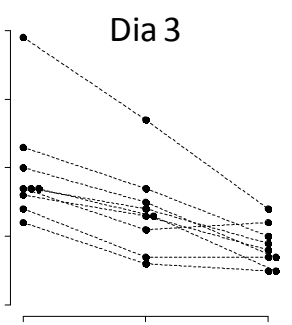

Figura 2. Número de visitas de abelhas Apis mellifera e frequência de comportamento de refugo, em que abelhas abandonam repentinamente flores antes de acessar os nectários, em flores Ludwigia tomentosa durante três dias consecutivos, no Pantanal do Rio Negro. Veja o desenho experimental nas lendas da Tabela 1 eFigura 1. Cada ponto representa uma flor e as linhas ligam os pontos de cada bloco diferente.

Figure 2. Number of visits of the bees Apis mellifera on flower of Ludwigia tomentosa and frequency of refuse behavior in which bees suddenly abandoned the flowers without accessing the nectaries, along three consecutive days in Pantanal of Rio Negro. See the experimental design in Table 1 and Figure 1 legends. Each point represents a flower and the lines connect the points to each block. 
2011) e a consequência dessas interações multitróficas pode resultar em maior ou menor sucesso reprodutivo da planta, pois aranhas que forrageiam sobre flores predam os visitantes florais, polinizadores ou não (Dukas 2001; Dukas \& Morse 2003; Suttle 2003).

Eventos onde o predador altera o sucesso da planta em que forrageia podem ser revertidos pela própria planta. Se a camuflagem é dependente da percepção das presas em detectar o predador, alterações fenotípicas nas plantas, tais como, mudanças na cor de suas pétalas, alteram o pano de fundo, o que consequentemente reduz a eficiência da camuflagem e reduz a predação sobre os polinizadores (Abbott 2010).

A estreita relação entre predador, planta e seus polinizadores apresentada neste trabalho, corrobora com a hipótese de que predadores, tal como aranhas, retiram polinizadores dos sistemas plantas-polinizadores (Romero \& Koricheva 2011). Mais estudos que contemplem tanto outros ecossistemas quanto outras espécies de predadores, plantas e visitantes florais são necessários para ampliar a compreensão de interações indiretas e, principalmente, entender os fatores que levam a variações nos resultados dessas relações.

Agradecimentos: N.Y. Kaku-Oliveira, C.M. Ferreira e C.G. Leal, por sua ajuda na execução do experimento, o Curso Ecologia do Pantanal e seus organizadores pela logística e aos dois revisores anônimos que contribuíram com o manuscrito. Coordenação de Aperfeiçoamento de Nível Superior (CAPES) pela bolsa concedida a autora N. Carvalho e ao Conselho Nacional de Desenvolvimento Científico e Tecnológico (CNPq) pela bolsa concedida a M. Delatorre.

\section{BIBLIOGRAFIA}

Аввотт, KR. 2010. Background evolution in camouflage systems: A predator-prey/pollinator-flower game. Journal of Theoretical Biology, 262:662-678.

CнітTKA, L. 2001. Camouflage of predatory crab spiders on flowers and the colour perception of bees. Entomological Genetics, 25:181-187.

Defrize, J; T Marc \& C JérôMe. 2010. Background colour matching by a crab spider in the field: a community sensory ecology perspective. The Journal of Experimental Biology, 213:1425-1435.

Dicke, M \& P Grostal. 2001. Chemical detection of natural enemies by arthropods: an ecological perspective. Annual Review of Ecology and Systematics, 32:1-23.

DukAs, R. 2001. Effects of perceived danger on flower choice by bees. Ecology Letters, 4:327-333.

DUKAS, R \& DH MORSE. 2003. Crab spiders affect flower visitation by bees. Oikos, 101:157-163.

DuKas, R \& DH Morse. 2005. Crab spiders show mixed effects on flower visiting bees and no effect on plant fitness. Ecoscience, 12:244-247.

FreItAS, AVL \& PS OliveIRA. 1996. Ants as selective agents on herbivore biology: effects on the behaviour of a nonmyrmecophilous butterfly. Journal of Animal Ecology, 65: 205-210.

Gonçalves-Souza, T; PM Omena \& GQ Romero. 2008. Trait-mediated effects on flowers: artificial spiders deceive pollinators and decrease plant fitness. Ecology, 89:2407-2413.

HalaJ, J \& DH Wise. 2001. Terrestrial trophic cascades: how much do they trickle? American Naturalist, 157: 262-281.

Harris, MB; C Arcangelo; ECT Pinto; G Camargo; MB Ramos-Neto; ET AL. 2005. Estimativas de perda da área natural da Bacia do Alto Paraguai e Pantanal Brasileiro. Conservação Internacional, Campo Grande, MS.

HeIIING, AM;KCHENG \& ME HerberSTEIN. 2004. Exploitation of floral signals by crab spiders (Thomisusspectabilis, Thomisidae). Behavioral Ecology, 15:321-326.

Jones, EI \& A Dornhaus. 2011. Predation risk makes bees reject rewarding flowers and reduce foraging activity. Behavioral Ecology and Sociobiology, 65:1505-1511.

LeONARD, AS; A Dornhaus \& DR PAPAJ. 2010. Flowers help bees cope with uncertainty: signal detection and the function of floral Complexity. The Journal of Experimental Biology, 214:113-121.

Morse, DH. 2007. Predator upon a ower: life history and fitness in a crab spider. Harvard University Press. Pp. 392.

NESS, JH. 2006. A mutualism's indirect costs: the most aggressive plant bodyguards also deter pollinators. Oikos, 113:506-514.

Pотт, A \& VJ Ротт. 2000. Plantas aquáticas do Pantanal. $1^{\mathrm{a}}$ edição. Embrapa. Comunicação para a transferência de tecnologia, Corumbá, MS. Pp. 404.

R Development Core Team. 2012. R: A language and environment for statistical computing. R Foundation for Statistical Computing, Vienna, Austria. ISBN 3900051-07-0. www.R-project.org.

Rocha-Filho, LC \& IMP Rinaldi. 2011. Crab spiders (Araneae: Thomisidae) in flowering plants in a Brazilian "Cerrado" ecosystem. Brazilian Journal of Biology, 71: 359-364.

Romero, GQ \& J Vasconcellos-Neto. 2005. Spatial distribution and microhabitat preference of Psecas chapoda (Peckham\&Peckham) (Araneae, Salticidae). Journal of Arachnology, 33:124-134.

Romero, GQ \& J Koricheva. 2011. Contrasting cascade effects of carnivores on plant fitness: a meta-analysis. Journal of Animal Ecology, 80:696-704.

StoKs, R; MA McPeек \& JL Mitchell. 2003. Evolution of prey behavior in response to changes in predation regime: damselflies in fish and dragonfly lakes. Evolution, 57:574-585.

SuttLe, KB. 2003. Pollinators as mediators of top-down effects on plants. Ecology Letters, 6:688-694.

THÉRY, M \& J CASAS. 2002. Predator and prey views of spider camouflage. Nature, 415:133-133.

Wignall, AE; AM Heiling; K Cheng \& ME Herberstein. 2006. Flower symmetry preferences in honeybees and their crab spiders predators. Ethology, 112:510-518.

ZAR, J. 2009. Biostatistical analysis. 5th edition. PrenticeHall, London. Pp. 960. 